\title{
Evaluation of systolic and diastolic blood pressure, pulse rate and SPO2 levels pre and post dental extraction under local anesthesia
}

\author{
Rahul VC Tiwari ${ }^{1, *}$, M Sridhar $^{2}$, Ravi Teja Painam ${ }^{3}$, Rama Krishna Suvvari ${ }^{4}$, Heena Tiwari ${ }^{5}$ \\ ${ }^{1}$ Fellow, ${ }^{2}$ Professor, ${ }^{3} \mathrm{PG}$ Student, ${ }^{4} \mathrm{PG}$ Student, ${ }^{\mathbf{1}} \mathrm{Jubilee}$ Mission Medical College Hospital and Research Center, Thrissur, Kerala, ${ }^{2-}$ \\ ${ }^{4}$ Sibar Institute of Dental Sciences, Takkellapadu, Gunutur, Andhra Pradesh, ${ }^{5}$ Government Dental Surgeon, Makdi, Kondagaon, \\ Chhattisgarh, India
}

*Corresponding Author:

Email: drrahulvctiwari@gmail.com

\begin{abstract}
Aim: To evaluate the changes in blood pressure, pulse rate and $\mathrm{SpO} 2$ pre during and post extraction procedure under local anesthesia. Materials and Method: A total of 150 patients were included in the study. Pulse oximeter was placed to evaluate the parameters and changes were investigated before administering local anesthesia, during anesthesia, during extraction procedure and post extraction. Data was tabulated and statistical analysis was done using 20 SPSS software.

Results: Almost of the patients has increase in the systolic blood pressure and pulse rate during administration of local anesthesia and during extraction procedure. Very minimal change was observed in diastolic blood pressure and SpO2 levels.

Conclusion: Significant changes are noted in changes in systolic blood pressure and pulse rate pre administration of local anesthesia, during administration of local anesthesia, during extraction, and post extraction procedure. Diastolic blood pressure and SpO2 has no significant differences.
\end{abstract}

Keywords: Blood pressure, Pulse rate, SpO2, Dental extraction, Local anesthesia.

\section{Introduction}

Hemodynamic changes are well proved which occurs in our day to day life in circulatory system of the body. Certain enzymes and chemicals are produced which are beneficial for our physical mechanism. These chemicals have effects on most of the systems like circulatory, respiratory and neurosensory etc. ${ }^{1}$ The nervous regulation is rapid among all the mechanism involved in the regulation of the arterial blood pressure. ${ }^{2}$ When the blood pressure alters, the nervous system brings the pressure back to normal within few minutes although nervous mechanism is quick in action, it operates only for a short period and then it adapts to the new pressure. Hence, it is called short - term regulation. ${ }^{3}$ The nervous mechanism regulating the arterial blood pressure operates through the vasomotor system. Hypothalamus regulates the blood pressure by acting on the vasomotor centers. ${ }^{4}$ Stimulation of posterior and lateral hypothalamic nuclei increases arterial blood pressure and stimulation of pre optic area decreases the blood pressure. ${ }^{5}$

Pulse rate is speed of heart beat measured by the number of contraction of heart per minute. It may vary according to body's physical needs including the need to observe oxygen and excrete carbon dioxide. It is usually equal or close to the pulse measured at any peripheral points. It is usually measured in the radial artery when checked manually. Normal pulse rate ranges from 60 to 100 beats per minute. The increase in heart rate is called as tachycardia and decrease in the heart rate is termed as bradycardia. ${ }^{6}$

Respiration is a process by which oxygen is taken in and carbon dioxide is given out. The normal respiratory rate in adults is 12 to 16 per minute. Respiration is often classified into external and internal respiration. External respiration involves exchange of respiratory gases i.e. oxygen and carbon dioxide between lungs and blood. Internal respiration which involves exchange of gases between blood and tissues. Whenever there is compensation in the respiratory mechanism its effects is reflected on the respiratory rate either via tachypnea or apnea. $\mathrm{SpO} 2$ stands for the peripheral capillary oxygen saturation, an estimate of the amount of oxygen in the blood. More specifically, it is the percentage of oxygenated hemoglobin compared to the total amount of hemoglobin in the blood. $\mathrm{SpO} 2$ is an estimate of arterial oxygen saturation, which refers to the amount of oxygenated hemoglobin in the blood. Hemoglobin is a protein that carries oxygen in the blood. It is found inside red blood cells and gives them their red colour. It is measured with the help of a pulse oximeter. Normal $\mathrm{SpO} 2$ value vary between 95 and $100 \%{ }^{7}$

The utility of this study will help the clinicians to empathize the basic knowledge about variations in vitals while performing routine dental extractions which can be helpful to manage the cases properly without any complications.

\section{Materials and Method}

Sample size: A hospital-based study was conducted using convenience sampling technique on 150 patients including 66 males and 84 females which were recruited from department of oral and maxillofacial surgery in SIBAR Institute of dental sciences, Guntur from $18 / 04 / 2016$ to $04 / 06 / 2016$. The minimum age was 27 years and maximum age was 83 years with the mean age of 54.42 years (Fig. 1). A random sampling technique was used for collection of samples. Data was collected and imported in Microsoft excel 2013 and analysis of data was 
done using Statistical Package for the Social Sciences (SPSS) ver. 20. Descriptive statistical analysis was performed. (Table 1)

Inclusion and exclusion criteria: All the patients with no relevant past medical history were included in the study and systemically compromised individuals and patients allergic to local anesthetics were excluded from the study Procedure: Each patient has been evaluated for blood pressure, respiratory disorders and random blood sugar level prior to inclusion. Informed consent has been taken from every subjects. All the procedures were performed by a single surgeon starting from patient counselling, administrating the local anesthetics, removal of tooth and post-operative instruction and medications to remove the bias in the study. A pulse oximeter (Larsen and Toubro Private Limited, India) was attached to the patient after the subjects gets seated on to the dental chair. Lidocaine $2 \%$ with 1:80,000 epinephrine is administered. (LIGNOX 2\% A, Promoted by Warren, Indoco Remedies limited) Extraction procedure is performed and after 5 minutes the pulse oximeter was removed. At four occasions the systolic and diastolic blood pressure, pulse rate and $\mathrm{SpO} 2$ were noted from the pulse oximeter i.e. pre-operative or before administrating local anesthetics, after administration of local anesthetics, during the extraction procedure and after the procedure is completed.

\section{Results}

Changes in systolic blood pressure: The minimum and maximum blood pressure before giving local anesthetic was 100 and 180 respectively with a mean of $130.70 \mathrm{~mm}$ of hg. After administration of local anesthetics the maximum value reached to 200 and minimum to 110 with the mean of $136.48 \mathrm{~mm}$ of $\mathrm{hg}$. During the extraction procedure the minimum value was 100 and maximum value was 180 with the mean value of $130.90 \mathrm{~mm}$ of $\mathrm{hg}$. After completion of the procedure the minimum value was 110 and maximum value was 200 with the mean value of 137.09. (Chart 1)

Changes in diastolic blood pressure: The minimum and maximum blood pressure before giving local anesthetic was 60 and 103 respectively with a mean of $81.84 \mathrm{~mm}$ of hg. After administration of local anesthetics the maximum value reached to 110 and minimum to 50 with the mean of $82.58 \mathrm{~mm}$ of hg. During the extraction procedure the maximum value was 103 and minimum value was 60 with the mean value of $82.49 \mathrm{~mm}$ of hg. After completion of the procedure the maximum value was 110 and minimum value was 50 with the mean value of 82.58 (Chart 2)

Changes in the pulse rate: The minimum and maximum pulse rate before giving local anesthetic was 68 and 121 respectively with a mean of 89.74 beats per minute. After administration of local anesthetics the maximum value reached to 122 and minimum to 72 with the mean of 98.39 beats per minute. During the extraction procedure the maximum value was 134 and minimum value was 72 with the mean value of 104.82 beats per minute. After completion of the procedure the maximum value was 134 and minimum value was 72 with the mean value of 103.78 (Chart 3)

Changes in the $\mathrm{SpO} 2$ :

The minimum and maximum $\mathrm{SpO} 2$ before giving local anesthetic was 94 and 100 respectively with a mean of $97.76 \%$. After administration of local anesthetics the maximum and minimum value were same but the mean increased to $98.29 \%$. During the extraction procedure the maximum value was 100 and minimum value was 90 with the mean value of $97.60 \%$. After completion of the procedure the maximum value was 100 and minimum value was 96 with the mean value of $97.73 \%$. (Chart 4)

\section{AGE DISTRIBUTION}

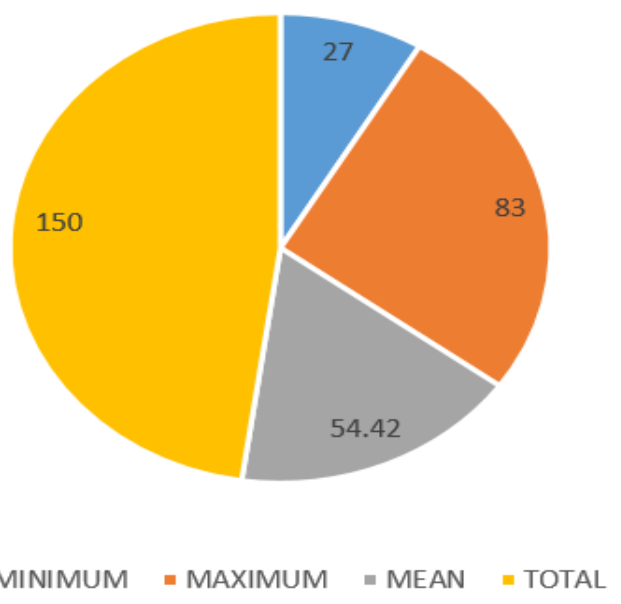

Fig. 1: Pie diagram showing distribution of subjects according to age

Table 1: Data showing the minimum, maximum and mean values of systolic and diastolic Blood Pressure, Pulse rate and Spo2

\begin{tabular}{|c|c|c|c|c|c|c|c|c|c|c|c|c|c|}
\hline \multirow{3}{*}{$\begin{array}{l}\text { S. } \\
\text { No. }\end{array}$} & \multirow[t]{3}{*}{ Procedure } & \multicolumn{6}{|c|}{ Blood pressure } & \multirow{2}{*}{\multicolumn{3}{|c|}{ Pulse rate }} & \multirow{2}{*}{\multicolumn{3}{|c|}{ SPO2 }} \\
\hline & & \multicolumn{3}{|c|}{ Systolic } & \multicolumn{3}{|c|}{ Diastolic } & & & & & & \\
\hline & & Min & Max & Mean & Min & Max & Mean & Min & Max & Mean & Min & Max & Mean \\
\hline 1 & Before L.A & 100 & 180 & 130.70 & 60 & 103 & 81.84 & 68 & 121 & 89.74 & 94 & 100 & 97.76 \\
\hline 2 & After L.A & 110 & 200 & 136.48 & 50 & 110 & 82.58 & 72 & 122 & 98.39 & 94 & 100 & 98.29 \\
\hline 3 & $\begin{array}{l}\text { During } \\
\text { Extraction }\end{array}$ & 100 & 180 & 130.90 & 60 & 103 & 82.49 & 72 & 134 & 104.82 & 90 & 100 & 97.60 \\
\hline 4 & $\begin{array}{l}\text { Post } \\
\text { Extraction }\end{array}$ & 110 & 200 & 137.09 & 50 & 110 & 82.58 & 72 & 134 & 103.78 & 96 & 100 & 97.73 \\
\hline
\end{tabular}


Chart 1: Showing minimum, maximum and mean values of systolic blood pressure SYSTOLIC BLOOD PRESSURE

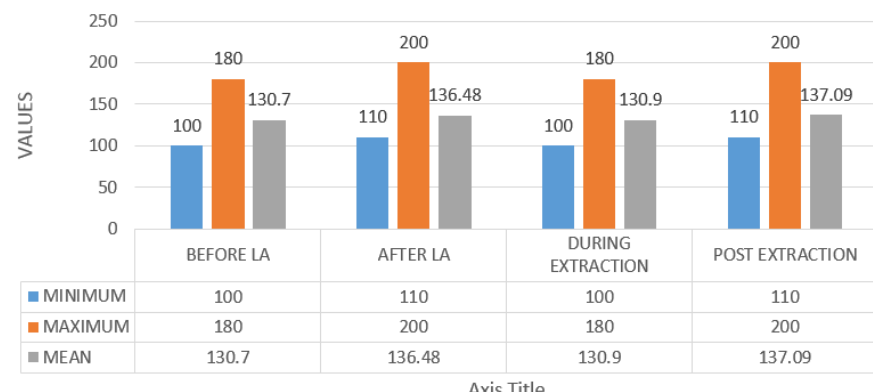

-MINIMUM $\square$ MAXIMUM $\square$ MEAN

Chart 2: Showing minimum, maximum and mean values of diastolic blood pressure

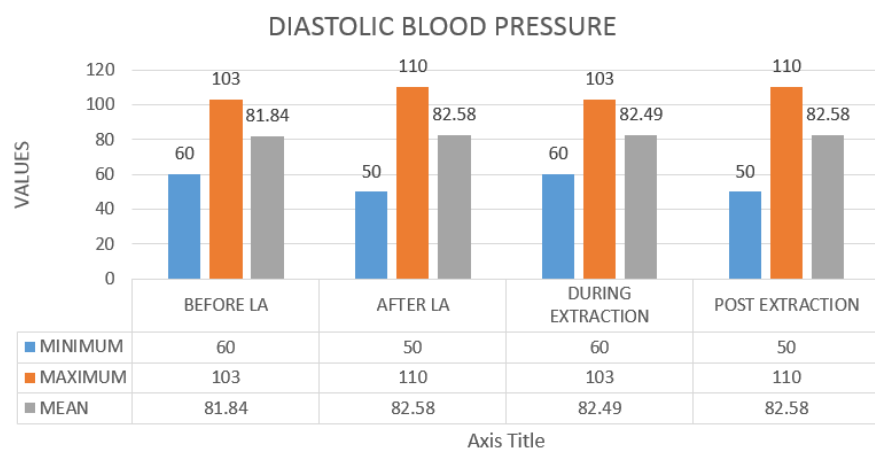

$\because$ MINIMUM $\square$ MAXIMUM $\because$ MEAN

Chart 3: Showing minimum, maximum and mean values of pulse rate

PULSE RATE

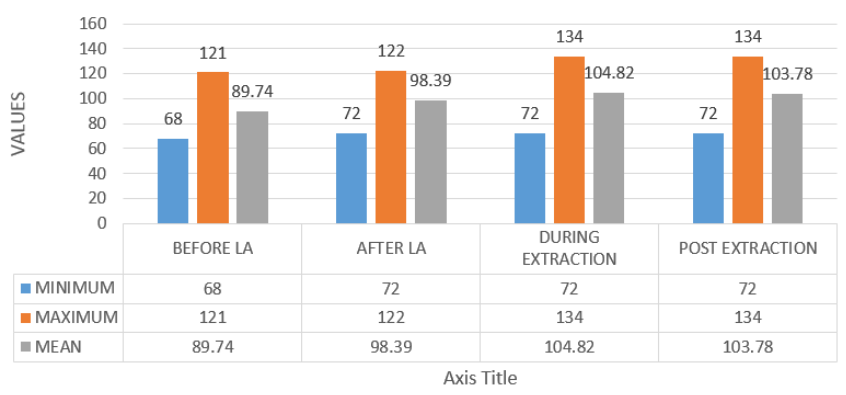

- MINIMUM $\because$ MAXIMUM $\because$ MEAN

Chart 4: Showing minimum, maximum and mean values of Spo2

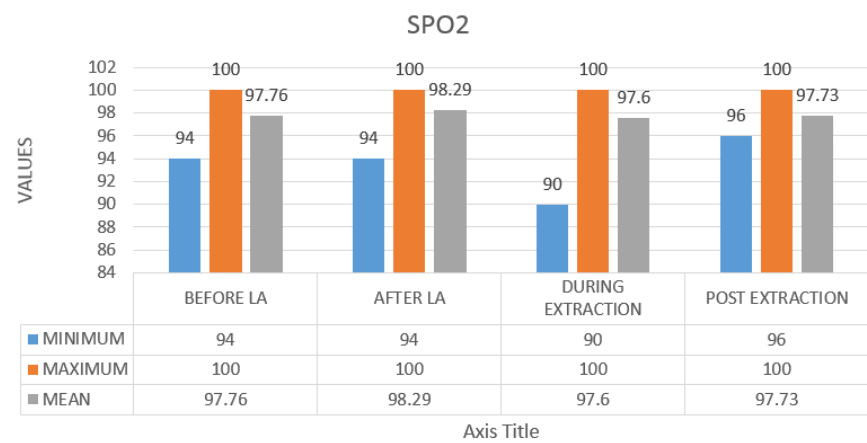

- MINIMUM MAXIMUM M MEAN 


\section{Discussion}

Lidocaine hydrochloride is an anesthetic agent which stabilizes the neuronal membrane by inhibiting the ionic fluxes required for the initiation and conduction of impulses thereby effecting local anesthetic action. Excessive blood levels cause changes in the cardiac output, total peripheral resistance, and mean arterial pressure. With central neural blockade these changes may be attributable to block of autonomic fibres, a direct depressant effect of the local anesthetic agent on various components of the cardiovascular system, and/or the beta-adrenergic receptor stimulating action of epinephrine when present. The net effect is normally a modest hypotension when the recommended dosages are not exceeded. This anesthetic agent comes in a mixture with epinephrine for its obvious advantages. Epinephrine more commonly known as adrenaline, is a hormone secreted by the medulla of the adrenal glands. Strong emotions such as fear or anger cause epinephrine to be released into the blood stream which causes increase in the heart rate, muscle strength, blood pressure and sugar metabolism. The reaction known as the flight or fight response prepares the body for strenuous activity. It is used chiefly as a stimulant in cardiac arrest, as a vasoconstrictor in shock and as a bronchodilator and antispasmodic in bronchial asthma. Epinephrine is found in small amounts in the body and is essential for maintaining cardiovascular hemostasis because of its ability to divert blood to tissues under stress. ${ }^{8}$

Whenever there is any surgical procedure to be performed, the patient goes into a state of fear which causes stress. Our body produces a surge of hormones when we are in a stressful situation. These hormones temporarily increase our blood pressure by causing our heart to beat faster and our blood vessels to narrow. These hormones have a regulatory effect on the blood pressure, heart rate and pulse rate. ${ }^{7}$ During the procedure of removal of tooth or dental extraction we administer local anesthetics prior to procedure with the help of syringes to give patient a painless and stress less experience. These local anesthetics contain epinephrine. During the procedure the patient goes into state of fear by looking at the armamentariums or fear of getting pain or any other outer, inner or environmental factors which causes stress. These all factors combine and play some or the other effect on the body systems. ${ }^{9}, 10$

Usually in hypertensive patients we avoid using local anesthetics with adrenaline as it can worsen the condition by increasing the heart rate and blood pressure which can lead to cardiac arrest and can be fatal. There are several studies which suggest that there is no difference in the hemodynamic changes in the body when compared using lidocaine with adrenaline and lidocaine without adrenaline in various minor oral surgical procedure. ${ }^{11,12}$ The response of blood pressure not only depends on the adrenaline based local anesthetics but it also depends upon various other factors like the type of dental surgery performed, the operating surgeons etc. ${ }^{1}$ Some studies suggests that there are significant changes in systolic blood pressure when compared to diastolic blood pressure when performing various dental procedures. ${ }^{13,14}$

A study on blood pressure levels pre and post extraction period was conducted on 100 Iraqi patients which have concluded that there was no significant differences in the systolic and diastolic blood pressure levels. ${ }^{15}$ Our results are contrast to this study. We have found that there are significant differences in the value of systolic blood pressure pre and post administration of local anesthetics and also pre and post extraction status. There is $5.78 \%$ increase in the systolic blood pressure pre and post administration of local anesthetics and 6.19\% increase pre and post extraction. The diastolic blood pressure had very minimal change which is not significant. Pulse rate has also a significant increase in the pre and post local anesthesia administration i.e. $8.65 \%$ and $6.43 \%$ increase pre and post extraction procedure. $\mathrm{SpO} 2$ had no significant increase or decrease pre and post-operative procedure. Some studies have given the similar results as ours in which authors have suggested that the systolic blood pressure shows marked increases of 3-24mm Hg during the extraction. ${ }^{16}$

In some studies highest increase in blood pressure are observed during complicated extractions in a hospital setting. The differences in increases of blood pressure cannot be attributed to age, gender or baseline blood pressure. ${ }^{17}$ However, the increase in blood pressure during extraction shows a relation with the dose of local anesthetic. Immediately after completion of the extraction, the increased systolic blood pressure begins to decline. ${ }^{18}$ The systolic blood pressure remains above the initial values for at least $10-15 \mathrm{~min}$ after completion of the extraction. According to some investigators, this increase can continue for up to 3 hour. ${ }^{19}$

A prospective randomized clinical trial was conducted on 120 patients to evaluate diastolic blood pressure, heart rate and $\mathrm{SpO} 2$ changes following injection of local anesthetics with different concentrations of epinephrine during simple tooth extraction and found no significant difference among the various local anesthetic agent like lidocaine $2 \%$ with epinephrine 1:80,000, articaine $4 \%$ with epinephrine and articaine $4 \%$ with epinephrine $1: 200,000 .{ }^{20}$ Lidocaine without and with a vasoconstrictor changes the heart rate and blood pressure differently. ${ }^{21}$ In another study it was found that the mean baseline blood pressure and pulse rate were $121 / 70 \mathrm{~mm} \mathrm{Hg}$ and 70 beats/min, respectively among the study subjects After the administration of local anesthetic (2\% lidocaine) containing 1:80,000 epinephrine, both the blood pressure and pulse rate increased. During dental surgery, blood pressure increased further to $132 / 73 \mathrm{~mm} \mathrm{Hg} .{ }^{22}$ Our analysis also shows the same results of increased blood pressure and pulse rate after the administration of local anesthetics and more increase during the dental extraction procedure. 


\section{Limitations of the study}

The study has been conducted on purely healthy patients and we have not included any medically compromised patient especially with cardiovascular diseases which is of more concern in our routine dental extraction procedure. The time when the procedure is performed in a day and for how much time the procedure is performed has not been taken into consideration including the waiting time of the patient in outpatient department.

\section{Conclusion}

Studies have indicated that cases of hypertension (above 200/100 $\mathrm{mm}$ of $\mathrm{Hg}$ ) are contraindicated for extraction and diastolic blood pressure has more variations when compares to systolic blood pressure. In our study we found significant changes in systolic blood pressure and pulse rate pre administration of local anesthesia, during administration of local anesthesia, during extraction, and post extraction procedure. Diastolic blood pressure and $\mathrm{SpO} 2$ has no significant differences. So we conclude that local anesthetic with adrenaline is safe to administer for extraction procedures in mild to moderate hypertensive cases.

\section{References}

1. Nakamura Y, Matsumura K, Miura K, Kurokawa H, Abe I, Takata Y. Cardiovascular and sympathetic responses to dental surgery with local anesthesia. Hypertens Res 2001;24:209-14.

2. Miura K, Matsumura K, Nakamura Y, Kurokawa H, Kajiyama M, Takata Y. Suppression of cardiac sympathetic nervous system during dental surgery in hypertensive patients. Hypertens Res 2000;23:207-12.

3. Matsumura K, Miura K, Takata Y, Kurokawa H, Kajiyama M, Abe I, et al. Changes in blood pressure and heart rate variability during dental surgery. Am J Hypertens 1998;11:1376-80.

4. Tsuchihashi T, Takata Y, Kurokawa H, Miura K, Maruoka Y, Kajiyama M et al. Blood pressure response during dental surgery. Hypertens Res 1996;19:189-94.

5. Sembulingam, K., \& Sembulingam, P. (2010). Essentials of medical physiology, $\left(5^{\text {th }}\right.$ ed. $)$, Jaypee Brothers Medical Publishers.

6. Barrett, K. E., Barman, S. M., Boitano, S., \& Brooks, H. L. (2010). Ganong's Review of Medical Physiology, $\left(23^{\text {rd }}\right.$ ed.), McGraw Hill Lange.

7. Colledge, N. R., Walker, B. R., \& Ralston, S. H. (2010). Davidson's Principles \& Practice of Medicine, $\left(21^{\text {st }} \mathrm{ed}\right.$.), Churchill Livingstone Elsevier.

8. Arthur C Guyton, John E Hall. (2011) Textbook of medical physiology. (12 ${ }^{\text {th }}$ ed.) Philadelphia, Pa: Saunders/Elsevier Publication.

9. Haas DA. An update on local anaesthetics in dentistry. J Can Assoc 2002; 68:546-51.

10. Sotunmbi PT, Arotiba JT. A manual of anesthesia for dental and maxillofacial surgery. $1^{\text {st }}$ ed. Ambod Publishers, Nigeria. 2005:72-102.

11. MO Ogunlewe'; O James ${ }^{\text {III }}$ J NA Ajuluchukwu ${ }^{\text {III; }}$ AL LadeindeI; WL Adeyemo'; OM Gbotolorun'. Evaluation of haemodynamic changes in hypertensive patients during tooth extraction under local anaesthesia. West Ind Med J 2011:60(1)
12. Gungormus M, Buyukkurt MC. The evaluation of changes in Blood pressure and pulse rate of hypertensive patient during Tooth Extraction. Acta Med. Austriaca 2003;30:127-9.

13. Nagao H, Munakata M, Tachikawa N, Shiota M, Kasugai S. Clinical study of risk management for dental implant treatment - changes of blood pressure and pulse rate during implant surgery under local anesthesia. Kokubyo Gakkai Zasshi 2002;69:27-33.

14. Brand H, Gortzak R, Palmer-Bouva C, Abraham R, Abraham-Inpijn L. Cardiovascular and neuroendocrine responses during acute stress induced by different types of dental treatment. Int. Dent J 1995;45(1):245-52.

15. Dr. Faaiz Alhamdani. The effect of immediate preextraction period on blood pressure level (Prospective study on 100 Iraqi patients). Mustansiria $D J$ 2008:5(3);290-29.

16. Goldstein D S, Dionne R, Sweet J, et al. Circulatory, plasma catecholamine, cortisol, lipid, and psychological responses to a real-life stress (third molar extractions): effects of diazepam sedation and of inclusion of epinephrine with the local anaesthetic. Psychosom Med 1982;44:259-72.

17. Meyer F-U. Haemodynamic changes under emotional stress following a minor surgical procedure under local anaesthe-sia. Int J Oral Maxillofac Surg 1987;16:688-94.

18. Meiller T F, Overholser C D, Kutcher M J, et a/. Blood pressure fluctuations in hypertensive patients during oral surgery. J Oral Maxillofac Surg 1983;41:715-8.

19. Tsuchihashi T, Takata Y, Kurokawa H, et a/. Blood pressure response during dental surgery. Hypertens Res 1996;19:189- 94.

20. Nedal Abu-Mostafa 1, Fatimah Al-Showaikhat 2, Fatimah Al-Shubbar 2, Kawther Al-Zawad 2, Fatimah Al- Banawi. Hemodynamic changes following injection of local anesthetics with different concentrations of epinephrine during simple tooth extraction: A prospective randomized clinical trial. J Clin Exp Dent 2015;7(4):e471-6.

21. FRITZ-ULRICH MEYER. Haemodynamic changes under emotional stress following a minor surgical procedure under local anaesthesia. Int J Oral Maxillofac Surg 1987:16:688-94.

22. Kiyoshi Matsumura, Keiko Miura, Yutaka Takata, Hideo Kurokawa, Minoru Kajiyama, Isao Abe, Masatoshi Fujishima. Changes in blood pressure and heart rate variability during dental surgery. AJH 1998;11:1376-80. 